\title{
PRECIPITADOS CERÁTICOS ATÍPICOS EM UM PACIENTE COM HANSENÍASE VIRCHOWIANA: RELATO DE CASO
}

\author{
ATYPICAL KERATIC PRECIPITATES INA LEPROMATOUS LEPROSY PATIENT: CASE REPORT
}

Jayter S. Paula ${ }^{1}$, Tarciso Schimrbeck², Felipe P. P. Almeida ${ }^{3}$, Erasmo Romão¹.

\begin{abstract}
${ }^{1}$ Docentes. ${ }^{2}$ Pós-graduando. ${ }^{3}$ Médico residente. Setor de Uveítes. Departamento de Oftalmologia, Otorrinolaringologia e Cirurgia de Cabeça e Pescoço. Faculdade de Medicina de Ribeirão Preto - USP.

Correspondência: Prof. Dr. Jayter Silva de Paula. Departamento Oftalmologia, Otorrinolaringologia e Cirurgia de Cabeça e Pescoço. Av. Bandeirantes, ํㅜ 3900, 12. andar. CEP: 14.049-900 - Ribeirão Preto - São Paulo, Brasil. Telefone: (16) 602-2499/ Fax: (16) 602-2860 E-mail: jayterdepaula@yahoo.com.br
\end{abstract}

Paula JS, Schimrbeck T, Almeida FPP, Romão E. Precipitados ceráticos atípicos em um paciente com hanseníase virchowiana: relato de caso. Medicina (Ribeirão Preto) 2005; 38(2): 150-155.

RESUMO: Modelo de Estudo: Relato de caso.

Importância do problema: A hanseníase é uma afecção que conduz a inúmeras alterações nos globos e anexos oculares, primordialmente no segmento anterior do olho, com relativa alta prevalência em países subdesenvolvidos. A iridociclite e as alterações corneanas provocadas cronicamente por essa infecção são as manifestações mais freqüentemente envolvidas no estabelecimento da cegueira.

Comentários: O caso descrito mostra um achado atípico do acometimento do segmento anterior do olho pela Hanseníase. À biomicroscopia observou-se precipitados ceráticos ovalados, acastanhados, distribuídos na metade inferior do endotélio corneano com aproximadamente $2 \mathrm{~mm}$ de diâmetro. Essas características ímpares, ainda não foram descritas na literatura. Após tratamento houve recuperação da acuidade visual do paciente, com manutenção dos achados de córnea. São feitas considerações a respeito dos aspectos epidemiológicos e de exame oftalmológico revisados sobre Hanseníase.

Descritores: Hanseníase. Iridociclite. Infecções Oculares. Endotélio da Córnea.

\section{1- INTRODUÇÃO}

A hanseníase é uma doença infecciosa crônica causada pelo bacilo Mycobacterium leprae, um parasita intracelular obrigatório. É considerada endêmica em várias regiões do mundo, principalmente em países subdesenvolvidos ou em desenvolvimento ${ }^{1}$. A Organização Mundial da Saúde considera a doença eliminada como problema de saúde pública quando a prevalência é de menos de um caso entre 10.000 pessoas $^{2}$. Em 2003, o Brasil foi o segundo lugar em prevalência da doença, atrás apenas da Índia, e du- rante o ano de 2004, segundo o Ministério da Saúde, teve prevalência de 4,5 pacientes para cada 10.000 habitantes ${ }^{3}$.

Observa-se desequilíbrio de prevalência entre as regiões brasileiras. O Estado do Rio Grande do Sul e o de Santa Catarina têm considerado a doença eliminada, enquanto que o Estado do Mato Grosso tem prevalência de 21/10.000 habitantes ${ }^{3}$. Devido ao grande número de casos novos no mundo (600.000 ao ano), há pouca perspectiva de erradicação da doença a curto prazo $^{1}$, embora tenha ocorrido considerável diminuição da incidência de casos novos após o início da po- 
liquimioterapia nos anos $80^{4}$. Antes do advento da poliquimioterapia, a hanseníase era a terceira causa de cegueira no mundo ${ }^{5}$.

$\mathrm{O}$ bacilo se desenvolve em temperaturas inferiores a $37^{\circ} \mathrm{C}$, explicando sua localização preferencial nas partes mais frias do corpo como nariz, lobos de orelhas, testículos, troncos nervosos periféricos e o segmento anterior do olho ${ }^{1,6,7}$. Lesões oculares podem surgir antes, durante ou após a cura microbiológica $^{2,8}$, sendo que apenas $41 \%$ dos portadores têm conhecimento da possibilidade de envolvimento ocu$\operatorname{lar}^{9}$. As manifestações oculares da doença são menos comuns nos estágios iniciais ${ }^{6}$, sendo mais frequientes com o agravamento da doença sistêmica ${ }^{10}$.

Dentre os achados na câmara anterior da hanseníase, as alterações irianas são típicas, sendo a presença de sinais de iridociclite, tais como celularidade de câmara anterior e precipitados ceráticos granulomatosos, comuns. Pérolas irianas são consideradas patognomônicas ${ }^{1}$, podendo ocorrer também outras lesões como atrofias, nódulos, sinéquias e pápulas ${ }^{11}$. No presente trabalho descreve-se o caso de um paciente com hanseníase, diminuição da acuidade visual e presença de precipitados ceráticos endoteliais atípicos.

\section{2- APRESENTAÇÃO DO CASO}

Paciente do sexo masculino, 31 anos, mulato, encaminhado ao Ambulatório de Oftalmologia do Hospital das Clínicas da Faculdade de Medicina de Ribeirão Preto (HCFMRP) relatando discreta diminuição da visão em ambos olhos, há aproximadamente 15 dias. Reclamava, também, de discreta vermelhidão e dor ocular. Negava outros sintomas ou qualquer enfermidade ocular prévia. O diagnóstico de hanseníase virchowiana tinha sido estabelecido dois meses antes no ambulatório de dermatologia do HCFMRP. Há 30 dias estava usando tratamento quimioterápico constituído por dapsona $100 \mathrm{mg}$ por dia, clofazimina $50 \mathrm{mg}$ por dia, com uma dose adicional de $300 \mathrm{mg}$ uma vez por mês e rifampicina $600 \mathrm{mg}$ uma vez por mês, durante 12 meses.

Ao exame oftalmológico apresentava acuidade visual de 0,8 em ambos os olhos, sem correção. Os reflexos pupilares luminosos estavam ligeiramente diminuídos bilateralmente. O exame dos anexos oculares revelou madarose bilateral de supercílios e cílios, principalmente na região temporal, sem qualquer outra alteração palpebral (Figura 1).

Ao exame na lâmpada de fenda observou-se hiperemia perilímbica, diminuição da sensibilidade da córnea, sem ceratite superficial, em ambos olhos. No endotélio havia a presença de múltiplos precipitados ceráticos ovalados castanho-dourados, distribuídos na metade inferior da córnea, com tamanho aproximado de $2 \mathrm{~mm}$ de diâmetro (Figura 2 e 3 ).

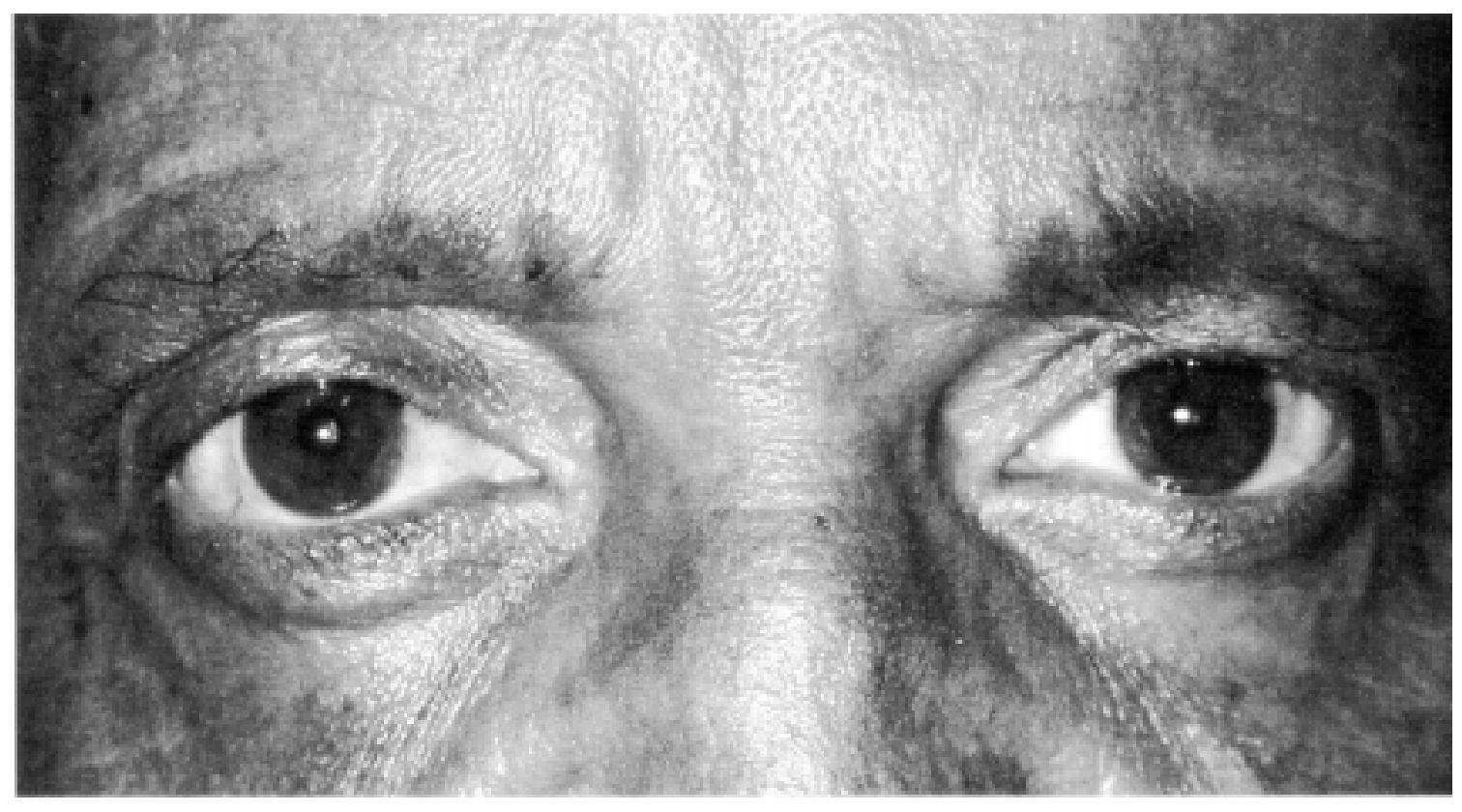

Figura 1 - Fotografia da face do paciente. Notar madarose bilateral de cílios e supercílios. 


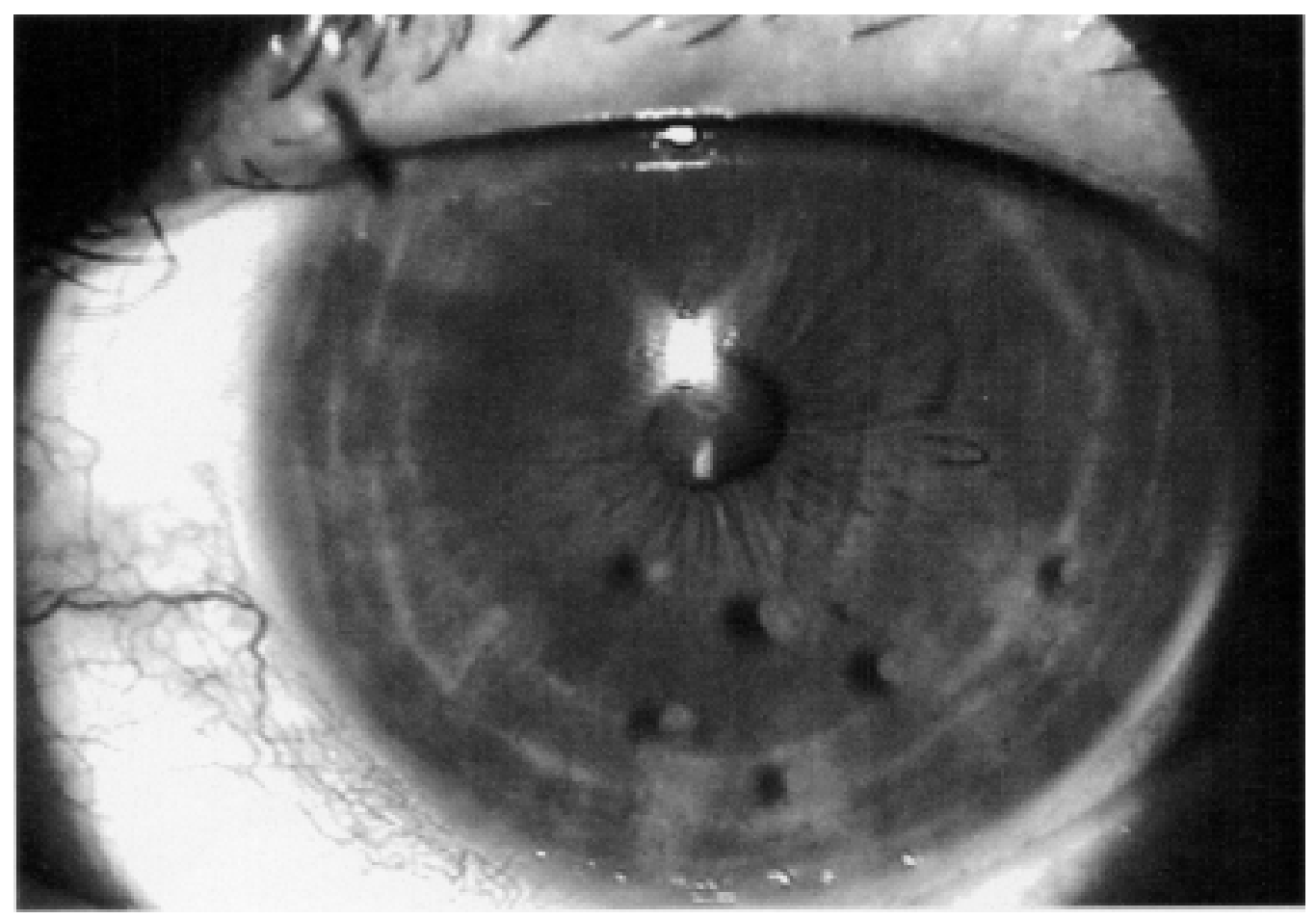

Figura 2 - Fotografia em lâmpada de fenda do olho esquerdo do paciente. Observar os múltiplos precipitados ceráticos ovalados, pigmentados, presentes na metade inferior da córnea.

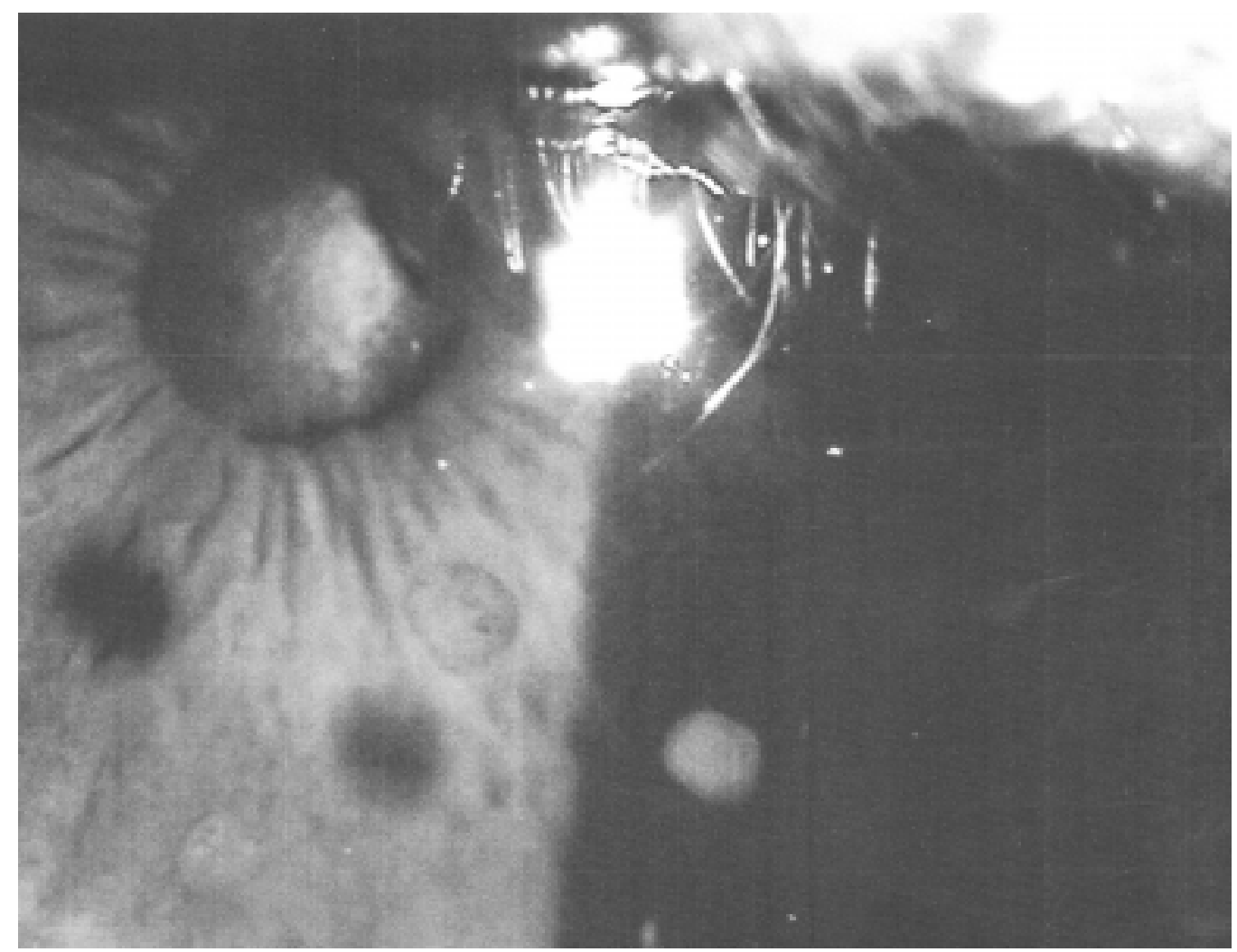

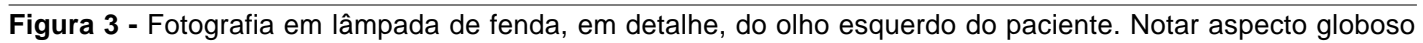
dos precipitados, aderidos ao endotélio corneano. 
Notou-se a presença de células inflamatórias em moderada quantidade na câmara anterior, sinéquias posteriores e pigmentos irianos na cápsula anterior do cristalino em ambos os olhos. Não foi constatada a presença de catarata e a pressão intra-ocular era $16 \mathrm{mmHg}$, em ambos os olhos. O paciente apresentou melhora da inflamação de câmara anterior após 15 dias de tratamento com colírio de dexametasona $0,001 \mathrm{~g} / \mathrm{ml}$, mantendo inalterados a acuidade visual e os demais achados. Após esse retorno, houve perda do seguimento clínico do paciente.

\section{3- DISCUSSÃO}

A avaliação oftalmológica de rotina em pacientes com hanseníase é importante, haja visto a variável frequiência (6 a 96\%) de alterações oculares ${ }^{1,10,12 / 22}$. Isto se deve a diferentes técnicas de exame utilizadas, examinadores não treinados, falta de instrumentos adequados e ao mal conhecimento das complicações oculares da doença ${ }^{10,23}$.

A hanseníase pode ser dividida em 4 grupos: forma precoce ou indeterminada, tuberculóide, virchowiana e limítrofe. A forma tuberculóide se caracteriza por lesões dermatológicas elevadas, únicas ou em pequeno número, e hipoestésicas ${ }^{4}$. O lagoftalmo é muito comum neste grupo ${ }^{1}$. Na forma virchowiana observa-se acometimento cutâneo difuso com distribuição simétrica ${ }^{4}$, sendo a madarose um achado oftalmológico típico ${ }^{1}$. Os portadores dessa forma da doença têm um risco duas vezes maior de sofrerem danos oculares que aqueles que apresentam a forma tuberculóide ${ }^{6,12,18,20}$.

As afecções corneanas acometem 10 a $20 \%$ dos pacientes com hanseníase ${ }^{6}$ e estão entre as mais freqüentes causas de cegueira (ceratite de exposição $21,3 \%$; opacidade corneana $13,5 \%$ ), acompanhadas de seqüelas de iridociclite crônica $(10,1 \%)$ e catarata complicada ${ }^{6}$. Estima-se que $1,3 \%$ a 3,2\% dos indivíduos com hanseníase sejam cegos ${ }^{24}$. Entretanto, um estudo no Nepal mostrou 9\% de cegueira, causada principalmente por uveítes e suas complicações (88\%), e por problemas corneanos em apenas $12 \%{ }^{2}$.

A falta de tratamento das lesões iniciais é a principal causa de perda visual ${ }^{1}$. A evolução das complicações oculares ocorre tanto nos indivíduos com a doença ativa, como naqueles que foram considerados curados por tratamento poliquimioterápico ${ }^{20,25}$. Geralmente, os pacientes são avaliados por oftalmologista somente nos casos sintomáticos ou quando apresentam perda visual acentuada.
Considera-se que a hanseníase afeta os olhos por meio de lesões causadas por reações inflamatórias aos antígenos do $M$. leprae e pela ação direta do bacilo ${ }^{1}$, embora exista estudo que discorde desta forma de acometimento ${ }^{7}$.

As alterações dos anexos oculares são as mais freqüentes, sendo a madarose de supercílios encontrada em $40 \%$, a de cílios em $33 \%$ e o lagoftalmo em $10 \%{ }^{1}$ dos pacientes com a forma virchowiana da doença ${ }^{26}$.

A catarata também é importante causa de cegueira na hanseníase, acometendo entre $15,2 \%$ a $24,0 \%$ dos pacientes ${ }^{6,10}$. Admite-se que esta se desenvolva pela uveíte, pelo uso de corticosteróide ou por invasão direta do bacilo ${ }^{1}$. O desenvolvimento do glaucoma relaciona-se à presença de sinéquias posteriores e goniossinéquias. As lesões no segmento posterior são pouco freqüentes e provocam pouca incapacidade visual quando comparadas às do segmento anterior. Vários autores descrevem lesões sugestivas da doença no segmento posterior ${ }^{26,27}$, inclusive com o relato de encontro do bacilo nessas áreas ${ }^{28}$. Alterações irianas na hanseníase acometem 7,2\% dos pacientes e não são associadas à diminuição da visão ${ }^{1,6,10}$. Além disso, com o advento da poliquimioterapia, a freqüência das uveítes vem se tornando cada vez menor, sendo observadas, quase que exclusivamente, na vigência de estados reacionais ${ }^{1}$.

As uveítes anteriores, causadas por invasão direta do bacilo, são geralmente insidiosas, assintomáticas, de extrema gravidade e as mais freqüentes. Entretanto, as uveítes secundárias a reações hansênicas são mais exuberantes, muito semelhantes a iridociclites anteriores agudas de outras afecções e, portanto, de mais fácil detecção ${ }^{1}$. Classicamente, os precipitados ceráticos encontrados na doença insidiosa são grumos de células inflamatórias que podem eventualmente se aglomerar com células epitelióides da íris, aderindo-se ao endotélio corneano, dando o aspecto característico da lesão ("mutton fat"). Tais lesões somente estarão presentes caso exista ou tenha existido uveíte no olho estudado $^{1,29}$.

O paciente descrito apresentava alterações oculares comumente observadas na hanseníase, tendo evoluído com melhora clínica, após o tratamento instituído para a iridociclite. Apesar da descrição da freqüente presença de precipitados ceráticos em pacientes portadores de iridociclite associada à hanseníase, inclusive do tipo granulomatoso, as lesões endoteliais observadas, com grandes e arredondados precipitados ceráticos, não haviam sido relatadas. Pela impossibilida- 
de de realização de biópsia das lesões, decorrente da perda do seguimento oftalmológico do paciente, especula-se se essas lesões poderiam corresponder aos grandes granulomas ou globias hansênicas de aparecimento raro, ou ainda a acúmulos de bacilos e monócitos, patologicamente semelhantes às pérolas irianas. Um estudo histopatológico futuro dessas lesões poderá elucidar a verdadeira origem das mesmas.

\section{4- COMENTÁRIOS FINAIS}

Apesar do advento da poliquimioterapia, a hanseníase continua sendo importante causa de envolvimento ocular e cegueira. O presente trabalho expõe a observação de precipitados ceráticos atípicos em um paciente com hanseníase tipo virchowiana, além de revisão epidemiológica dos achados oculares presentes nessa doença.

Paula JS, Schimrbeck T, Almeida FPP, Romão E. Atypical keratic precipitates in a lepromatous leprosy patient: case report. Medicina (Ribeirão Preto) 2005; 38(2): 150-155.

ABSTRACT: Paper design: Case report.

Clinical relevance: Leprosy can lead to many abnormalities in the eyes, mainly in the anterior chamber, with high prevalence in undeveloped countries. Chronic iridociclitis and corneal lesions are the most frequent ocular alterations associated with leprosy frequently causing blindness.

Comments: This report describes an atipical anterior chamber lesion caused by leprosy. Oval, about $2 \mathrm{~mm}$ brownish keratic precipitates, distributed in the inferior corneal endothelium, could be seen by slit lamp examination. These rare precipitates have not yet been described in the literature. The visual acuity of the patient was recovered after treatment, but the corneal alterations were still present. Epidemiological and ophthalmological considerations about Hansen disease were also reviewed.

Keywords: Leprosy. Iridocyclitis. Eye Infections. Endothelium, Corneal.

\section{REFERÊNCIAS BIBLIOGRÁFICAS}

1 - Campos WR, Rodrigues CAF, Oréfice F. Hanseníase. In: Oréfice F. Uveíte: clínica e cirúrgica. Rio de Janeiro: Editora Cultura Médica; 2000. vol.1 p. 341-66.

2 - Combate à hanseníase ganha reforço 10/11/2004. Disponível em: http://portal.saude.gov.br/saude/aplicacoes/noticias/ noticias_detalhe.cfm?co_seq_noticia=11913. [2004 nov 13].

3 - World Health Organization Leprosy Elimination Project Status, Report 2003. Disponível em: http://www.who.int./lep/Reports/ s20042.pdf. [2004 nov 13].

4 - Miller RA. Hanseníase (Lepra). In: Fauci AS, Braunwald E, Isselbacher KJ, Wilson JD, Martin JB, Kasper SL et al eds. Harrison medicina interna. $14^{\mathrm{a}}$ ed. Rio de Janeiro: Editora Mc Graw Hill; 1998. p. 1085-90.

5 - Johnstone PA, George AD, Meyers WM. Ocular lesions in leprosy. Ann Ophthalmol 1991; 23: 297-303.

6 - Ffytche TJ. The prevalence of disabling ocular complications of leprosy: a global study. Indian J Lepr 1998; 70: 49-59.

7 - Ebenezer GJ, Ebenezer D. Pathology of a lepromatous eye. Int J Lepr 2000; 68: 23-6.
8 - Lewallen S, Tungpakorn NC, Kim SH, Courtright P. Progression of eye disease in "cured" leprosy patients: implications for understanding the pathophysiology of ocular disease and for addressing eyecare needs. Br J Ophtahlmol 2000; 84: 817-21.

9 - Yowan P, Danneman K, Koshy S, Richard J, Daniel E. Knowledge and practice of eye-care among leprosy patients. Indian J Lepr 2002; 74: 129-35.

10 - Daniel E, Koshy S, Rao GS, Rao PS. Ocular complication in newly diagnosed borderline lepromatous and lepromatous leprosy patients: baseline profile of the Indian cohort. $\mathrm{Br} \mathrm{J}$ Ophthalmol 2002; 86: 1336-40.

11 - Oréfice F, Campos WR. Hanseníase. In: Oréfice F. Uveítes. São Paulo: Editora Roca; 1987. p. 265-72.

12 - Toríbio R. Incapacidade visual em hanseníase. Brasília Méd 1999; 36: 118.

13 - Khan T, Awan AA, Kazmi HS, Shah AA, Muhammad S. Frequency of ocular complications of leprosy in institutionalized patients in NWFP Pakistan. J Ayub Med Coll Abbottabad 2002; 14: 29-33. 
14 - Mvogo CE, Bella-Hiag AL, Ellong A, Achu JH, Nkeng PF. Ocular complications of leprosy in Cameroon. Acta Ophthalmol Scand 2001; 79: 31-3.

15 - Monteiro LG, Campos WR, Oréfice F, Grossi MA. Study of ocular changes in leprosy patients. Indian J Lepr 1998; 70: 197-202.

16 - Lubbers WJ, Schipper A, Hogeweg M, De Soldenhoff R. Eye disease in newly diagnosed leprosy patients in eastern Nepal. Lepr Rev 1994; 65: 231-8.

17 - Sekhar GC, Vance G, Otton S, Kumar SV, Stanley JN, Rao GN. Ocular manifestations of Hansen's disease. Doc Ophthalmol 1994; 87: 211-21.

18 - Mvoso SN, Nvoso MC. Ocular findings in leprosy patients in Nigeria. East Afr Med J 1994; 71: 441-4.

19 - Dana MR, Hochman MA, Viana MA, Hill CH, Sugar J. Ocular manifestations of leprosy in a noninstitutionalized community in the United States. Arch Ophthalmol 1994; 112: 626-9.

20 - Nepal BP, Shrestha UD. Ocular findings in leprosy patients in Nepal in the era of multidrug therapy. Am J Ophthalmol 2004; 137: 888-92.

21 - Soshamma G, Suryawanshi N. Eye lesions in leprosy. Lepr Rev 1989; 60: 33-8.

22 - Kagme GK. Ocular leprosy in Africa. Soc Sci Med 1983; 60: 33-8.

23 - Courtright PD. Defining the magnitude of ocular complications from leprosy: problems of methodology. Int $\mathrm{J}$ Lepr Other Mycobact Dis 1988; 17: 566-73.
24 - Lamba PA, Santoshkumar D, Arthanariswaran R. Ocular leprosy - a new perspective. Leprosy in India 1983; 55: 490-4.

25 - Courtright P, Hu LF, Li HY, Lewallen S. Multidrug therapy and eye disease in leprosy: a cross-sectional study in the People's Republic of China. Comment In. Int J Epidemiol 1994; 23: 835-42.

26 - Moreno RD, Woods W, Moreno N, Trindade R, Neto JT. Alterações oculares na hanseníase, observadas em pacientes ambulatoriais do serviço de referência da cidade de Rio Branco, Acre-Brasil. Arq Bras Oftalmol 2003; 66: 755-64.

27 - Monteiro LG, Campos WR, Oréfice F, Grosi MAF. Estudo das alterações em hansenianos de controle ambulatorial. Rev Bras Oftalmol 1992; 51: 43-6.

28 - Oréfice F, Miranda D, Boratto LM. Encontro de bacilos de Hansen na conjuntiva, corpo vítreo e retina em um olho enucleado de paciente portador da forma virchowiana. Arq Bras Oftalmol 1990; 53: 17-9.

29 - Meira DM, Oréfice F. Sinais e sintomas nas uveítes. In: Oréfice F. Uveíte: clínica e cirúrgica. Rio de Janeiro: Editora Cultura Médica; 2000. vol.1 p. 15-6.

Recebido para publicação em 22/11/2004

Aprovado para publicação em 21/03/2005 